\title{
Influence of bacterial burden on meibomian gland dysfunction and ocular surface disease
}

This article was published in the following Dove Press journal:

Clinical Ophthalmology

\author{
Alanna Nattis' \\ Henry D Perry ${ }^{2,3}$ \\ Eric D Rosenberg ${ }^{4}$ \\ Eric D Donnenfeld ${ }^{5}$ \\ 'Department of Ophthalmology, \\ Lindenhurst Eye Physicians and Surgeons, \\ P.C., Babylon, NY I I702, USA; \\ ${ }^{2}$ Department of Ophthalmology, Nassau \\ University Medical Center, East Meadow, \\ NY I I554, USA; ${ }^{3}$ Department of \\ Ophthalmology, Ophthalmic Consultants \\ of Long Island, Rockville Centre, NY \\ II 570, USA; ${ }^{4}$ Department of \\ Ophthalmology, New York Medical \\ College, Valhalla, NY 10595, USA; \\ ${ }^{5}$ Department of Ophthalmology, \\ Ophthalmic Consultants of Long Island, \\ Garden City, NY I I530, USA
}

Purpose: Bacterial burden on the eyelid margin and within meibomian glands was evaluated for influence on specific ocular surface disease (OSD) markers across the meibomian gland dysfunction (MGD) spectrum.

Methods: In this prospective, observational, single-center study, 40 patients were divided into 4 equal groups of 10 that encompassed increasingly worse MGD/OSD categories. All patients answered the standard Ocular Surface Disease Index questionnaire, and underwent tear osmolarity testing (TOT), Schirmer 1, matrix metalloproteinase 9 (MMP-9) testing, meibography, and lissamine green staining. Cultures of eyelid margins and meibomian gland secretions were directly plated on blood, chocolate, and Sabouraud agar; smears were sent for gram and Papinicolau evaluation.

Results: Mean patient age was $55.25 \pm 17.22$ years; there were 10 males and 30 females. TOT and MMP-9 testing were similar across groups. Culture positivity was $62.5 \%$ for right eyes, $70 \%$ for left eyes, and was not statistically different across groups (for both eyelid margin and meibomian glands). The majority of cultures were positive for coagulasenegative staphylococcus (CNS).

Conclusion: This study is in concordance with others, citing the predominance of CNS within the biofilm of both "normal" and clinically significant MGD/OSD patients. Our study exemplifies that symptoms of OSD do not necessarily correlate with degree of clinical exam findings, nor culture positivity. These results argue that bacterial burden should be reconsidered as a direct risk factor and treatment target for MGD/OSD patients.

Keywords: blepharitis, biofilm, dry eye, meibomian gland dysfunction, ocular surface disease

\section{Introduction}

Meibomian glands are modified sebaceous glands that are arranged vertically in the tarsal plate. ${ }^{1}$ With each blink, meibum is released and interacts with the tear film to create a smooth refractive surface and maintain consistent quality of vision. ${ }^{1}$ Patients with meibomian gland dysfunction (MGD) suffer visual consequences secondary to tear film instability, reduced tear break up time and evaporative dry eye. ${ }^{1}$ In the early stages of MGD, patients may be asymptomatic - but if left untreated, it will either cause or exacerbate dry eye symptoms (dryness, burning, itching, foreign body sensation, photophobia, tearing, intermittent blurred vision). ${ }^{1}$

MGD, blepharitis, and dry eye disease are overlapping and challenging entities to treat, secondary to lack of a single evident treatment target. Multiple studies have linked imbalance in the bacterial flora with blepharitis and MGD - however, it is unclear if the bacteria cause MGD, if MGD allows for bacterial overgrowth, or if
Department of Ophthalmlogy,

Lindenhurst Eye Physicians and Surgeons,

P.C., 500 W Main Street Suite 210,

Babylon, NY I I702, USA

Tel + I 63I 9573355

Email ASN5I6LU@gmail.com 
specific factors released from the bacteria (eg, lipases) lead to meibomian gland inspissation, plugging and disruption of normal gland and eyelid physiology. ${ }^{1-14}$ In this light, antibiotics, specifically macrolides (eg, azithromycin, erythromycin), fluoroquinolones and tetracyclines (eg, doxycycline) have been studied for their efficacy and mechanism of action in treating MGD. ${ }^{1,3,4,5,8-11}$ In concert with this, studies have demonstrated the predominant organisms that live on the eyelids. Presence of coagulase-negative staphylococcus (CNS) (eg, Staphylococcus epidermidis), Staphylococcus aureus, Corynebacterium, and Propionibacterium acne, has been delineated in "normal" patients and those with blepharitis. ${ }^{8,9,10,15-20}$

Slit lamp examination and use of vital dyes, such as lissamine green have historically allowed for delineation of the extent of ocular surface disease (OSD). Additionally, the ophthalmologist has an armamentarium of diagnostic tests available to evaluate dry eye - whether evaporative or aqueous-deficient in nature. For example, there is testing available for the inflammatory marker matrix metalloproteinase-9 (MMP-9) presence in the tears, suggesting that these patients with positive tests may respond favorably to anti-inflammatory therapy. ${ }^{6}$ High tear osmolarity levels suggest aqueous deficiency. ${ }^{6,20}$ Meibography may also be used for evaluation of patients with MGD/dry eye, as meibomian gland loss (“drop-out") has been significantly correlated to dry eye and evaporative tear film dysfunction. ${ }^{12,20}$ In concert with this, Schirmer 1 testing may be used to evaluate aqueous tear deficiency. ${ }^{20}$

We now know that various resident species of bacteria on the eyelids may be implicated in either the etiology or propagation of MGD. ${ }^{8,9,10,15-19}$ To date, no studies have been performed evaluating the correlation of bacterial burden on specific dry eye/OSD markers (ie, tear osmolarity, Schirmer tests, meibography, or MMP-9 positivity). These tests, in conjunction with slit lamp examination and corneal staining, are important indicators of the type of dry eye a patient has, and the direction in which treatment should be guided. In addition, comparison of eyelid/meibomian gland bacterial burden across disease spectrum (ie, normal, asymptomatic, subclinical, and clinically significant meibomian gland dysfunction patients) on the forementioned diagnostic parameters has not been described. In completion of this study, the goals were to establish relationships between bacterial culture positivity (of eyelid margin, meibomian glands) and degree of OSD and MGD as elucidated on clinical exam and by a variety of in-office diagnostic tests.

\section{Methods}

Before commencement of the study, study protocols, consent forms, and data accumulation methods were approved by the Institutional Review Board of NuHealth (Nassau University Medical Center, East Meadow, NY). Written informed consent was obtained from each patient at screening and Health Insurance Portability and Accountability Actregulations were followed. The study was conducted according to the tenets of the Declaration of Helsinki.

This was a prospective observational study comprised of 40 patients ( 80 eyes) in a single practice setting. The study was comprised of four equal groups (“A,B,C,D”) of patients, who underwent evaluation for signs and symptoms of dry eye and meibomian gland dysfunction. Inclusion criteria were 18 years old or older, and the ability to consent for eye exam, including routine diagnostic procedures related to dry eye evaluation. Exclusion criteria were pregnancy, inability to consent, age $<18$ years old, active ocular infection, and current use of antibiotic and/or antifungal medications (topical or systemic). The examined patients were assigned to one of the four groups based upon Ocular Surface Disease Index (OSDI) score and degree of meibomian gland disease present on examination.

Group A served as controls. The majority of these patients' visits were for routine eye exams without frank evidence of dry eye and/or MGD on exam. These patients had an OSDI score of 0-40. Group B were "asymptomatic" patients with little-to-no complaints of eye discomfort but had exam components suggestive of mild dry eye/MGD, with an OSDI score of 50 or below. Group C were those patients who had "subclinical disease". These patients had occasional complaints consistent with OSD (eg, dryness, irritation, redness) with evidence of mild-moderate dry eye and/or MGD on exam, as well as an OSDI score of 63 or below. Group D consisted of patients with clinically significant meibomian gland dysfunction/OSD. These patients had symptoms of near-constant burning/irritation/dryness, etc., as well as evidence of significant disease (eg, meibomian gland plugging, dropout, positive dry eye markers) on exam, and an OSDI score of 100 or below.

\section{Evaluation}

Each patient in the study had both eyes evaluated. Patient complaints were recorded and all underwent standard slit lamp examination. All patients had cultures and sensitivities 
of their eyelid margin and meibomian gland secretions. All participants answered a standard OSDI Questionnaire ${ }^{(21}$

In addition to the questionnaire, each subject underwent comprehensive evaluation of past medical/ocular history. Uncorrected (UCVA) and best-corrected (BCVA) visual acuity was measured using the Snellen chart. Comprehensive slit lamp examination was performed, noting the presence and grade of meibomian gland dysfunction, drying of the ocular surface, eyelid irregularities, and tear breakup time. Grading of meibomian gland dysfunction (ease of expression and type of secretions) was performed according to the International Workshop on Meibomian Gland Dysfunction. ${ }^{5}$

Tear osmolarity testing (TOT) was performed using the standard TearLab $^{\circledR}$ (San Diego, CA, USA) system. MMP9 testing was performed using the standard Inflammadry ${ }^{\circledR}$ (Rapid Pathogen Screening, Sarasota, FL, USA) system. Schirmer I testing was performed in the standard manner (ie, without anesthetic) for both eyes. Meibography was performed to evaluate meibomian gland status/dropout using the Oculus Keratograph ${ }^{\circledR}$ system (Oculus, Arlington, WA, USA). Comparison of dropout in the right upper and lower, as well as left upper and lower lids was performed across groups. Results were graded via the Meiboscale and degree of meibomian gland loss was converted into percentages for data analysis. ${ }^{19}$

Gradation of lissamine green staining of the conjunctiva and cornea was performed for each eye according to the Oxford Grading System (scale 0 through 4, with 0 equating to no staining and 4 meaning dense, confluent staining). Cultures of the eyelid margin were performed using platinum spatula by gently scraping the eyelid margin, and sent for evaluation on blood, Sabouraud, and chocolate agar plates. Meibomian gland secretions were obtained by applying pressure to the eyelid margin with two cotton-tipped applicators, one on the outer eyelid, one in the fornix, after application of a topical anesthetic. Secretions were plated directly from the cotton-tip applicator containing meibum onto the culture plates. Scraping from the eyelid margin and meibum from meibomian gland expression was directly applied to a glass cytology slide, which was immediately fixed in methanol. These slides were sent for gram stain and cytology studies.

Primary outcome measures were degree of bacterial burden (ie, culture positivity as well as sensitivity studies) and culture results as correlated with dry eye and meibomian gland dysfunction diagnostic parameters.

Secondary outcome measures were comparison of culture and testing results between eyes, comparison of culture and testing results across the spectrum of dry eye/meibomian gland dysfunction, comparison of OSDI questionnaire responses with exam findings, testing and culture results, as well as analysis of demographic variables (eg, age, sex) on culture results, OSDI responses, exam findings and diagnostic testing results.

\section{Statistical considerations}

Study variables were analyzed by appropriate statistical methods to assess for the differences among the four groups. Correlation between testing parameters, meibomian gland dropout, ocular surface staining, culture, gram stain and cytology positivity between groups, and demographics were assessed using ANOVA, chi-square $\left(\chi^{2}\right)$, Student's $t$ test, and the Fisher z-transformation test. $p$-values less than or equal to 0.05 were considered statistically significant.

\section{Results \\ Overall findings and correlation with culture positivity}

Forty patients from October 2016 through March 2017 were evaluated in a single practice setting. Mean patient age was $55.25 \pm 17.22$ years (range 18-81); there were 10 males and 30 females. When evaluating age in respect to results, the patients in the control group were younger $(40.7 \pm 12.27$ ); this was statistically significant (ANOVA, $p=0.014)$.

All patients completed an OSDI survey. Overall mean OSDI score was $36.16 \pm 19.45$ (range 6-75). As shown in Figure 1, mean OSDI scores were increasingly worse from Group A (19.73 \pm 8.43$)$ to B (31.355 \pm 7.81$)$, to C (39.08 $\pm 11.586)$ to $\mathrm{D}(54.46 \pm 15.054)$; this was statistically significant (ANOVA; $p<0.001)$. OSDI score across groups was not statistically correlated with positive lid ( $t$-test, $p=0.686$ OD and $p=0.463 \mathrm{OS}$ ) or meibomian gland ( $t$-test, $p=0.769$ OD and $p=0.765$, respectively) cultures.

Mean TOT OD was $302.05 \pm 13.59$; OS was 301.5 \pm 15.18 . TOT score was not statistically significant across groups or eyes (ANOVA, $p=0.977$ and $p=0.819$, respectively). When comparing TOT results versus eyelid margin culture positivity (OD and OS), no statistical relationship was found ( $t$-test, $p=0.630$ and $p=0.673$, respectively). No statistical relationship was found with TOT results and meibomian gland culture positivity (OD or OS) ( $t$-test, $p=0.873$ and $p=0.87$, respectively)

Thirty-seven and one-half percent of right eyes were MMP-9 positive; $47.5 \%$ of left eyes were MMP-9 
OSDI Score

by group

A
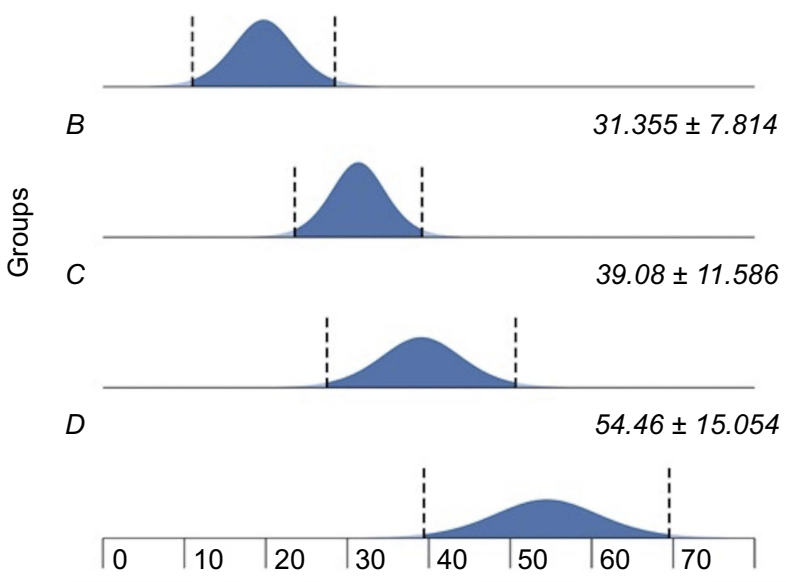

OSDI

Score

Figure I Increasing Ocular Surface Disease Index (OSDI) scores by group (ANOVA; $p<0.001$ ).

positive; however, positive results were not statistically different across groups or eyes $\left(\chi^{2} p=0.205 ; p=0.659\right.$, respectively). MMP-9 positivity was not statistically correlated to positive eyelid (z-score, $p=0.722$ OD and $p=0.191$ OS) or meibomian gland (z-score, $p=0.752$ OD and $p=0.288$ OS) cultures.

Average Schirmer 1 score for OD was $16.53 \pm 15.18$ (range 0-35); and 16.38 \pm 9.19 (range 0-35) OS. Schirmer 1 scores were not statistically significantly different across groups or eyes (ANOVA, $p=0.113, p=0.200$, respectively). There was no statistical relationship found between Schirmer 1 scores and eyelid margin ( $t$-test, $p=0.081$ and $p=0.845$ ) or meibomian gland cultures ( $t$-test, $p=0.052$, $p=0.790$ ) in right and left eyes, respectively.

Mean percentage meibomian gland dropout on meibography was $31.5 \pm 17.26$ for the right upper lid (RUL), 38.63 \pm 22.42 for the right lower lid (RLL), $31.5 \pm 18.21$ for the left upper lid (LUL) and 39.39 \pm 18.03 for the left lower lid (LLL). Difference in gland dropout was not statistically different across groups for the RUL (ANOVA, $p=0.062$ ); however, it was statistically different across groups for the RLL (ANOVA, $p=0.005$ ), with increasing amounts of dropout from Group A through Group D. This is demonstrated in Figure 2. Percentage gland loss was also statistically different and worsened across Group A through Group D for the left upper and lower eyelids (ANOVA, $p=0.026$ and $p=0.032$, respectively). These findings are shown in Figures 3 and 4 ,
Meibography RLL by group

A

$28.5 \pm 13.174$

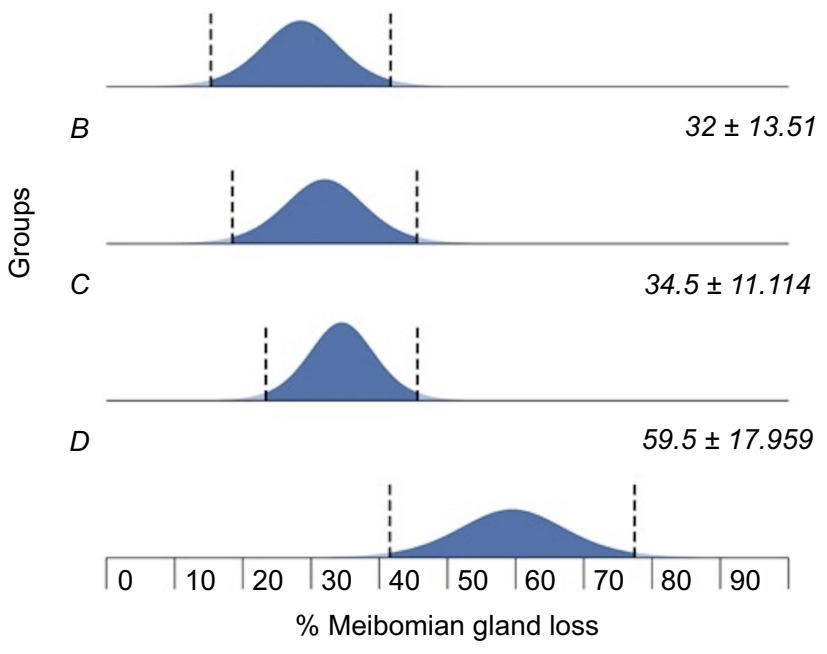

Figure 2 Increasing meibomian gland loss in right lower lid (RLL) by group (ANOVA; $p=0.005$ ).

respectively. Percentage meibomian gland loss was not statistically correlated to positive eyelid margin ( $t$-test, $p=0.0498)$ or meibomian gland ( $t$-test, $p=0.451)$ cultures.

Mean overall lissamine green staining score for the conjunctiva was $1.2 \pm 0.84$ for the right eye and $1.29 \pm 1.03$ for the left eye. Conjunctival lissamine green staining across all groups was increasingly (and significantly) worse from Group A to D. As shown in Figures 5 and 6, respectively, this was true for both right (ANOVA, $p=0.004$ ) and left (ANOVA, $p=0.041)$ eyes. Conjunctival lissamine green staining was not statistically correlated with positive eyelid margin ( $t$-test, $p=0.338$ OD and $p=0.070$ OS) or meibomian gland ( $t$-test, $p=0.669$ OD and $p=0.079$ OS) cultures.

Mean corneal lissamine green score for the right eye was $0.4 \pm 0.46$ (range $0-2$ ) and $0.45 \pm 0.49$ (range $0-2$ ), and was not statistically different across all groups (ANOVA, $p=0.679$ [OD], $p=0.726$ [OS]). Corneal lissamine green staining was not statistically correlated with positive eyelid margin ( $t$-test, $p=0.829$ OD and $p=0.781$ OS) or meibomian gland ( $t$-test, $p=0.395$ OD and $p=0.756$ OS) cultures.

Mean grade of meibomian gland secretions was 3.02 \pm 1.13 for the right eye and $3.01 \pm 1.11$ for the left eye. Gradation of meibomian gland secretions was not statistically correlated with positive eyelid margin ( $t$-test, $p=0.198$ OD and $p=0.732$ OS) or meibomian gland ( $t$ test, $p=0.925$ OD and $p=0.612$ OS) cultures. 
Meibography LUL

by group

A

$18 \pm 9.266$

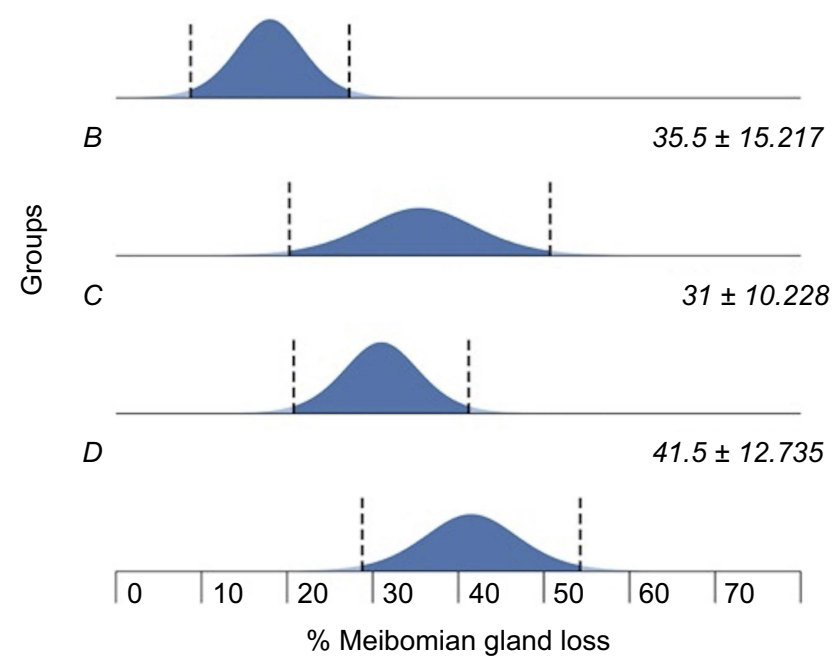

Figure 3 Increasing meibomian gland loss in left upper lid (LUL) by group (ANOVA; $p=0.026$ ).

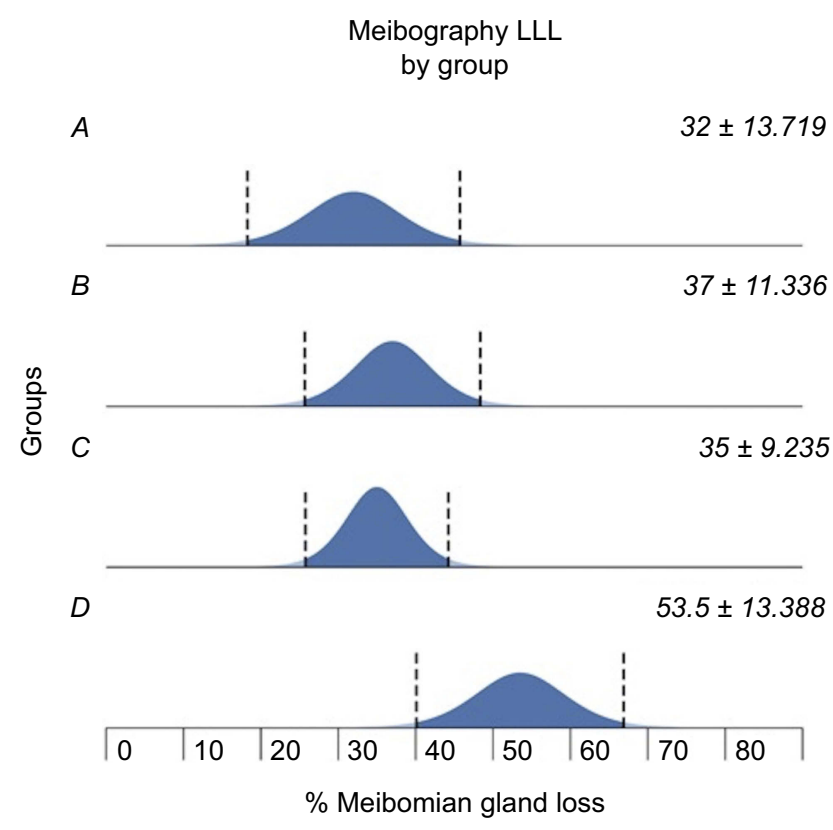

Figure 4 Increasing meibomian gland loss in left lower lid (LLL) by group (ANOVA, $p=0.032$ ).

Overall, there were 25 right eyes (62.5\%) and 30 left eyes $(75 \%)$ with positive cultures. Across groups and eyes, eyelid margin culture positivity was not statistically significant ( $\chi^{2} p=0.813$ and $p=0.439$, respectively). Additionally, across groups and eyes, meibomian gland culture positivity was not statistically significant $\left(x \chi^{2}\right.$ $p=0.380$ and $p=0.710$, respectively).
Conjunctival lissamine grade OD by group

A

$0.85 \pm 0.585$

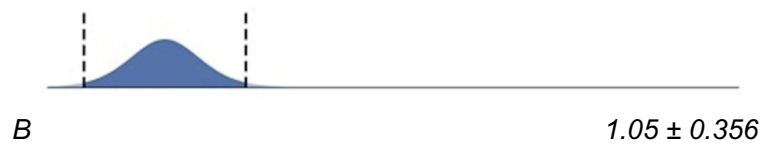

$$
\text { 음 }
$$
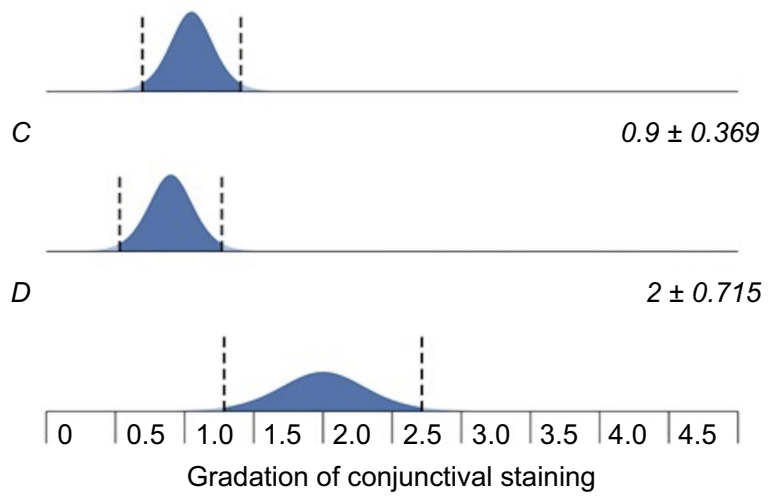

Figure 5 Increasing conjunctival staining across groups, right eye (ANOVA; $p=0.004$ ).

Conjunctival lissamine grade OS by group

A

$0.8 \pm 0.699$
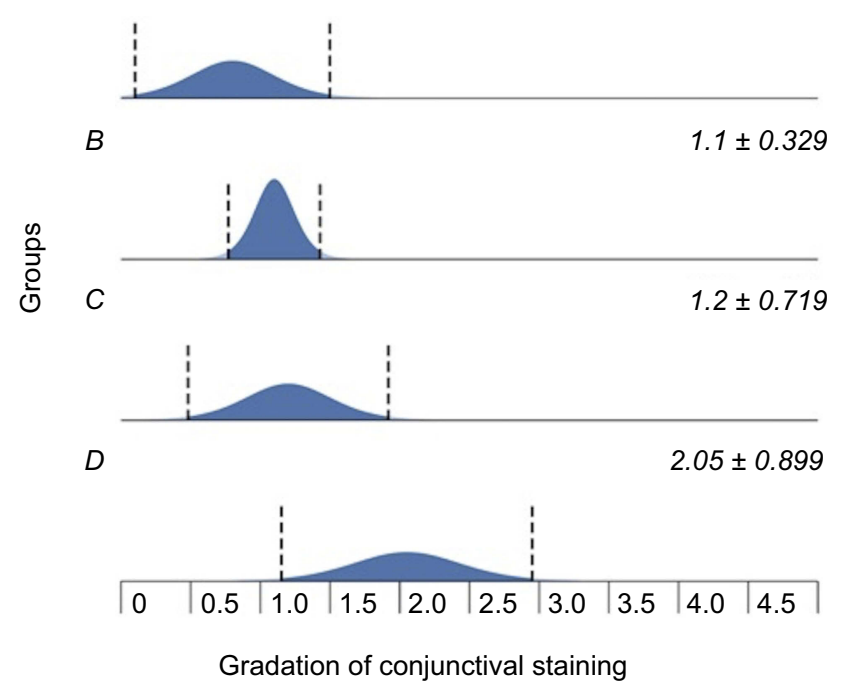

Figure 6 Increasing conjunctival staining across groups, left eye (ANOVA; $p=0.041$ ).

Of those patients with positive cultures, 12 right eyes (48\%) and 19 left eyes (63\%) had positive lid margin cultures. Of those patients with positive cultures, meibomian gland cultures were positive in 13 right eyes $(52 \%)$ and $11(36 \%)$ left eyes. Two eyes had positive gram stain (with positive correlation with culture results); no eye had positive cytology (Papinicolau) findings. Of all positive 
cultures, 21 right eyes (84\%) and 23 left eyes (76\%) were positive for CNS, ie, S.epidermidis). Of all positive cultures, one right eye (4\%) was resistant to erythromycin (CNS) and ten (33\%) left eye cultures were resistant to erythromycin (CNS, S. aureus). Of all positive cultures, most were susceptible to tetracycline. Two left eye cultures (6\%) were tetracycline resistant (both CNS).

\section{Results: group breakdown/specifics Group A results}

Group A consisted of "control patients" with little-no complaints of eye discomfort. Ten patients (20 eyes) comprised this group, with six male and four female patients. The average age was $40.7 \pm 16.3$ years; mean OSDI score was $19.73 \pm 11.6$. When comparing meibomian gland loss in Group A versus all other groups, there was statistically less gland dropout for the RUL (test, $p=0.026$ ) and LUL ( $t$ test, $p=0.006$ ). Meibomian gland secretions/expression scores were significantly better in Group A compared with other groups for both right ( $t$-test, $p=0.019$ ) and left eyes ( $t$-test, $p=0.011)$. Eyelid margin cultures were positive in $40 \%$ and $60 \%$ of right and left eyes, respectively. Meibomian gland secretions were positive in $50 \%$ and $40 \%$ of right eye left eyes, respectively. Three additional species aside from CNS were cultured (Corynebacterium species, Candida parapsilosis, and S. aureus). Diagnostic/ testing results for this group are displayed in Table 1.

\section{Group B results}

Group B consisted of ten (one male, nine female) patients (20 eyes) with average OSDI score $31.36 \pm 10.4$. Mean age was $61 \pm 12.8$ years. Thirty percent of right eyelid margin samples were culture positive; $40 \%$ of left eyelid margin samples were culture positive. Meibomian gland secretions were positive in $40 \%$ and $20 \%$ of right and left eyes, respectively. There were two right eye cultures positive for organisms other than CNS (Staphylococcus lugdunesis, S. aureus) and one left eye culture positive for S. aureus. Diagnostic/testing results for this group are shown in Table 2.

\section{Group C results}

Group C comprised the "subclinical meibomian gland disease" patients, with average OSDI score of $39.08 \pm 15.3$. This group had ten patients ( 20 eyes), two males and eight females; average age was $61.7 \pm 12.6$ years. Thirty percent and $60 \%$ of eyelid margin cultures were positive in right and left eyes, respectively. Meibomian gland cultures were positive in $20 \%$ of right eyes and $30 \%$ of left eyes. Three right
Table I Group A results

\begin{tabular}{|l|l|l|}
\hline Parameter & OD & OS \\
\hline TOT & $302.6 \pm 6.5$ & $304.9 \pm 18.58$ \\
\%MMP-9+ & 10 & 40 \\
Schirmer I score & $22.7 \pm 9.9$ & $20.8 \pm 9.6$ \\
Conjunctival LG score $^{\text {Corneal LG score }}$ & $0.85 \pm 0.78$ & $0.8 \pm 0.93$ \\
MG expression score $^{\mathrm{a}}$ & $0.25 \pm 0.6$ & $0.35 \pm 0.63$ \\
\% Total positive cultures & $2.3 \pm 1.27^{\mathrm{a}}$ & $2.25 \pm 1.17^{\mathrm{a}}$ \\
\% Positive lid margin cultures $^{\mathrm{b}}$ & 50 & 60 \\
\% Positive MG cultures $^{\mathrm{b}}$ & $90 \%$ & $100 \%$ \\
\% Positive CNS $^{\mathrm{b}}$ & $100 \%$ & $66 \%$ \\
\%Erythromycin resistance $^{\mathrm{b}}$ & 100 & 100 \\
\%Tetracycline resistance $^{\mathrm{b}}$ & 0 & 33 \\
\hline
\end{tabular}

Notes: a'Statistically significant results for MG Expression score. ${ }^{\text {b} P e r c e n t a g e ~ o f ~}$ culture positive group.

Abbreviations: MG, meibomian gland; OD, right eye; OS, left eye; LG, lissamine green.

Table 2 Group B results

\begin{tabular}{|c|c|c|}
\hline Parameter & OD & OS \\
\hline TOT & $303.3 \pm 18.4$ & $298.9 \pm 11.04$ \\
\hline \%MMP-9+ & 40 & 40 \\
\hline Schirmer I score & $15.8 \pm 10.8$ & $14.9 \pm 9.3$ \\
\hline Conjunctival LG score & $1.05 \pm 0.47$ & $1.1 \pm 0.4$ \\
\hline Corneal LG score & $0.5 \pm 0.55$ & $0.6 \pm 0.58$ \\
\hline MG expression score & $3.3 \pm 1.04$ & $3.4 \pm 0.92$ \\
\hline \%Total positive cultures & 50 & 50 \\
\hline$\%$ Positive lid margin cultures ${ }^{a}$ & 80 & 80 \\
\hline$\%$ Positive MG cultures ${ }^{\mathrm{a}}$ & 80 & 40 \\
\hline$\%$ Positive CNS ${ }^{\mathrm{a}}$ & 100 & 80 \\
\hline \%Erythromycin resistance ${ }^{a}$ & 0 & 30 \\
\hline$\%$ Tetracycline resistance ${ }^{a}$ & 0 & 10 \\
\hline
\end{tabular}

Note: ${ }^{2}$ Percentage of culture positive group.

Abbreviations: MG, meibomian gland; OD, right eye; OS, left eye; LG, lissamine green.

Table 3 Group C results

\begin{tabular}{|c|c|c|}
\hline Parameter & OD & os \\
\hline TOT & $300.6 \pm 15.03$ & $299.2 \pm 15.6$ \\
\hline \%MMP-9+ & 50 & 60 \\
\hline Schirmer I score & $|5| \pm 5.6$. & $17.9 \pm 6.33$ \\
\hline Conjunctival LG score & $0.9 \pm 0.5$ & $1.2 \pm 0.9$ \\
\hline Corneal LG score & $0.45 \pm 0.35$ & $0.4 \pm 0.4$ \\
\hline MG expression score & $3.2 \pm 0.7$ & $3.2 \pm 0.7$ \\
\hline$\%$ Total positive cultures & 40 & 70 \\
\hline$\%$ Positive lid margin cultures ${ }^{a}$ & 75 & 86 \\
\hline$\%$ Positive MG cultures $^{\mathrm{a}}$ & 50 & 43 \\
\hline$\%$ Positive CNS ${ }^{a}$ & 75 & 71 \\
\hline \%Erythromycin resistance ${ }^{a}$ & 10 & 30 \\
\hline$\%$ Tetracycline resistance ${ }^{a}$ & 0 & 10 \\
\hline
\end{tabular}

Notes: ${ }^{2}$ Percentage of culture positive group.

Abbreviations: MG, meibomian gland; OD, right eye; OS, left eye; LG, lissamine green. 
eye and two left eye cultures were positive for organisms other than CNS (Corynebacterium species, Acinetobacter calcoaceticus; and A. calcoaceticus, Haemophilus influen$z a e$, respectively). Diagnostic/testing results for this group are shown in Table 3.

\section{Group D results}

Group D comprised "clinically significant meibomian gland disease" patients with mean OSDI score of $54.5 \pm 19.96$. This group was composed of ten (one male, nine female) patients (20 eyes) with an average age of $56.7 \pm 17.29$ years. When comparing Group D meibography scores to all other groups combined, there was statistically greater percentage gland loss in Group D for the RLL ( $t$-test, $p<0.001)$, LUL ( $t$-test, $p=0.046$ ), and LLL ( $t$-test, $p=0.003$ ). Group D also had statistically worse conjunctival staining OD ( $t$-test, $p<0.001)$ and OS ( $t$-test, $p=0.006$ ). Meibomian gland drop out scores are shown in Table 5. In this group, $20 \%$ of right eyelid margin samples were culture positive; $30 \%$ of left eyelid margin cultures were positive. Meibomian gland cultures were positive in $20 \%$ of both right eye left eye samples. Two right eyes had cultures positive for organisms other than CNS (Actinomyces species, Corynebacterium species) and three left eyes had cultures positive for other organisms (Stenotrophomonas maltophilia, Pantoea agglomerans, Cladosporium species). Testing results from Group D are shown in Table 4.

\section{Discussion}

Treatment of meibomian gland dysfunction and OSD remains challenging, despite our array of diagnostic tests

Table 4 Group D results

\begin{tabular}{|l|l|l|}
\hline Parameter & OD & OS \\
\hline TOT & $301.7 \pm 11.4$ & $301.6 \pm 13.76$ \\
\%MMP-9+ & 50 & 60 \\
Schirmer I score & $12.5 \pm 8.5$ & $12.8 \pm 8.81$ \\
Conjunctival LG score $^{\mathrm{a}}$ & $2 \pm 0.95^{\mathrm{a}}$ & $2.05 \pm 1.9^{\mathrm{a}}$ \\
Corneal LG score & $0.4 \pm 0.2$ & $0.45 \pm 0.27$ \\
MG expression score $^{\text {\% Total positive cultures }}$ & $3.3 \pm 1.1$ & $3.2 \pm 1.17$ \\
\% Positive lid margin cultures & \\
\% Positive MG cultures & 40 & 30 \\
\% Positive CNS & 50 & 100 \\
\%Erythromycin resistance $^{\mathrm{b}}$ & 50 & 66 \\
\%Tetracycline resistance $^{\mathrm{b}}$ & 25 & 66 \\
\hline
\end{tabular}

Notes: ${ }^{a}$ Statistically significant results for conjunctival lissamine stain score. ${ }^{\mathrm{b}}$ Percentage of culture positive group.

Abbreviations: MG, meibomian gland; OD, right eye; OS, left eye; LG, lissamine green.
Table 5 Percentage meibomian gland loss across groups

\begin{tabular}{|l|l|l|l|l|}
\hline \%MG loss & RUL & RLL & LUL & LLL \\
\hline Group A & 21 & $28.5 \pm 17.5$ & $18 \pm 12.3^{\mathrm{a}}$ & $32 \pm 18.2$ \\
& $\pm 15.13^{\mathrm{a}}$ & & & \\
Group B & $36.5 \pm 20.9$ & $32 \pm 17.9$ & $35.5 \pm 20.2$ & $37 \pm 15.03$ \\
Group C & $29.5 \pm 10.9$ & $34.5 \pm 14.7$ & $31 \pm 13.6$ & $35 \pm 12.2$ \\
Group D & $40 \pm 13.78$ & 59.5 & 41.5 & 53.5 \\
& & $\pm 23.82^{\mathrm{a}}$ & $\pm 16.89^{\mathrm{a}}$ & $\pm 17.76^{\mathrm{a}}$ \\
\hline
\end{tabular}

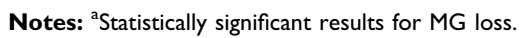

Abbreviations: MG, meibomian gland; RUL, right upper lid; RLL, right lower lid; LUL, left upper lid; LLL, left lower lid.

and ability to perform comprehensive in-office evaluations. As demonstrated in our results, varying culture positivity as well as a variety of different organisms was found within and between groups, regardless of symptoms and diagnostic testing scores. The majority of positive cultures were for CNS and $S$. aureus; their presence on the skin and eyelid margin has been well documented. ${ }^{1,5,9,15,16,33,34}$ Other organisms found in our study, such as Corynebacterium, Bacillus, Actinomyces, and $H$. influenzae have also been noted to be present on the lid margins, however to a much lesser extent compared to CNS and S. aureus. ${ }^{8,15,22-32,35}$

Our study demonstrated increasing OSDI scores as degree of meibomian gland dysfunction/OSD worsened which is not unexpected. Additionally, TOT, MMP-9, and Schirmer testing did not significantly change across the disease spectrum, but conjunctival lissamine green staining and degree of meibomian gland dropout (as shown on meibography) did deteriorate in the groups with clinically significant disease. We think that the degree of lissamine staining was in relation to severity of the evaporative dry eye (as demonstrated by meibomian gland loss) and thus paralleled increased symptomatology in these patients. This would be in agreement with the "dry eye blepharitis syndrome (DEBS)" theory as suggested by Rynerson and Perry, with dry eye, blepharitis and meibomian gland dysfunction existing as one disease condition on a continuous spectrum. ${ }^{15}$

Several studies have elaborated on the influence of the bacterial biofilm, bacterial lipases, and bacterial colonization on the eyelids and within meibomian gland secretions correlating with degree of meibomian gland dysfunction and OSD. ${ }^{6,7,9,15,17,18}$ Our study findings reinforce prior studies citing the prevalence of coagulase-negative staph on the lid margin; however, it did not elucidate any significant, nor definitive correlation between positive eyelid margin and meibomian gland cultures and degree of disease. ${ }^{6,7,8,9,15,17,18}$ This may in part be real or secondary to the relatively small sample size of our study. 
The culture positivity across the meibomian gland dysfunction spectrum is in concordance with the theory devised by Drs Rynerson and Perry, who suggested that dry eye and blepharitis are not distinct entities but rather a disease spectrum influenced by the biofilm that forms on the lid margin over time. ${ }^{9,15}$ We hypothesize that construction of this biofilm may in fact explain the results showing less meibomian gland dropout and superior meibomian gland expression scores in Group A versus all other groups. ${ }^{15}$ In addition, formation of a biofilm may have allowed for colonization with the unusual organisms seen in our study - a finding that, to our knowledge, has not been published previously. However, it is also important to note that even with good culture techniques, contamination is not an impossibility.

Throughout history, many treatments for dry eye, blepharitis, and MGD have consisted of antibiotic use (eg, erythromycin, azithromycin, doxycycline); the presence of a bacterial biofilm on the eyelid and within meibomian glands appears to be a reasonable treatment target. However, we are still unsure of the impact of the bacterial biofilm on disease severity and chronicity - is it the mere presence of bacteria, formation of bacterial lipases, invasion of meibomian glands by bacteria, and/or disruption in normal flora that is the culprit? Our study highlights and confirms the presence of bacteria (mainly CNS) across the dry eye/MGD spectrum, and most notably, we did not find any correlation between culture positivity, organism type, and dry eye diagnostic parameters. ${ }^{15-17}$

As it stands now, we are afforded many different options as far as treatment for dry eye - from artificial tears, to serum tears, to medications such as cyclosporine $0.05 \%$ (Restasis $^{\circledR}$, Allergan, Parsippany-Troy Hills, NJ, USA), compounded cyclosporine, lifitegrast $5 \%$ (Xiidra ${ }^{\circledR}$, Shire, Lexington, MA, USA), even intranasal neurostimulation (TrueTearTM, Allergan, Parsippany-Troy Hills, NJ, USA) ${ }^{5,15}$ Similarly, management of blepharitis may consist of warm compresses, eyelid hygiene using saline or medicated scrubs/sponges (eg, Avenova ${ }^{\circledR}$ [NovaBay Pharmaceuticals Inc. Emeryville, CA, USA], OCuSOFT ${ }^{\circledR}$ [OCuSOFT, Inc., Rosenberg TX, USA]), as well as electromechanical debridement using BlephEx ${ }^{\circledR}$ (BlephEx, Franklin, TN, USA). ${ }^{1,3,4-11,15,17,18}$ Adding to this is the nutritional treatment component of oral omega-3 supplementation. ${ }^{1,3-10,11,15,17,18}$ However, with all of these available treatments, as well as the new and evolving available diagnostics for OSD, it is challenging to find the best treatment algorithm for each patient. The patients, who are usually uncomfortable due to their condition, and frustrated secondary to lack of treatment efficacy, add to that challenge. Our study points out that this challenge is not easily met as signs or symptoms often do not reflect clinical findings and even cultural identification may be of little help. Anecdotally, the authors have noted some guidance from diagnostic tests such as MMP-9, Schirmer 1, and lissamine green staining. MMP-9 may be helpful in suggesting anti-inflammatory therapy. Schirmer 1 being 3 or less may argue for treatment with cyclosporine and/or punctal plugs or cautery. Lissamine green staining may also be beneficial for following patients and evaluating the effects of treatment.

Our results pose a challenge for development of a "universal" treatment algorithm for dry eye/MGD in terms of targeting the microbial milieu. One may argue MDG/OSD should be treated via eradication of the bacterial biofilm - this may be accomplished by mechanical debridement (eg, Blephex ${ }^{\circledR}$, meibomian gland expression, Lipiflow ${ }^{\circledR}$ [TearScience ${ }^{\circledR}$, Morrisville, NC, USA]) and/or antibiotic use (topical or oral). In our study, $20 \%$ of all cultures were found to be macrolide (erythromycin, specifically) resistant; whereas only $3.6 \%$ were tetracyclineresistant (all coagulase negative staph). We believe this information is meaningful in that we should rethink the treatment of blepharitis with antibiotics (specifically macrolides), and perhaps make use of doxycycline (if necessary), not necessarily for its antimicrobial effect, but anti-inflammatory effect on the eyelid margin and within meibomian glands. ${ }^{1,3,4,5,9,10}$

The biofilm created by bacteria, especially staphylococcal species (S. aureus and S. epidermidis in particularthe most prominent organisms seen in our study) is quite strong, and may be resistant to white blood cells, antibiotics, and povidone iodine scrubs. ${ }^{4,15}$ Additionally, it has been shown that the bacteria in the biofilm can release virulence factors (cytolytic toxins, proteases, and lipases capable of destroying host tissue) causing escalating levels of inflammation not only on the lid margin, but also on the ocular surface, meibomian glands, and accessory lacrimal glands. ${ }^{1,3,4,5,15,16,34,36}$ The biofilm likely thickens and diversifies with age, explaining why MGD and dry eye worsen with increasing age. ${ }^{4,5,11,12,15}$ If we cannot effectively eradicate this biofilm that resides on the eyelid margin, we cannot effectively reach the areas of the eye/ eyelids that require treatment.

Thus, perhaps our patients who are on the dry eyeblepharitis-meibomian gland dysfunction spectrum who 
are not improving on the "usual therapies" could benefit from mechanical eyelid debridement, essentially "wiping the slate clean" to increase the efficacy of therapies such as lid hygiene, moist heat, topical sprays, and cleansers. With its various presentations, it is easy to focus on the immediate presenting problem (eg, when a patient has meibomian gland disease, the lash follicles may be ignored; when a patient has lacrimal gland involvement, the meibomian glands may be overlooked). ${ }^{1,4,5,6,7,15,17,37}$ The omnipresence of bacteria on the eyelid margins and within meibomian glands is well known, and as we noted, does not appear to change in any particular direction across the disease spectrum..$^{1,2,3,5,7-11}$ Our data suggest that perhaps the most important aspect of the dry eye-meibomian gland dysfunction-blepharitis spectrum to recognize the presence, thickness and diversity of the bacterial biofilm in our patients - especially those with recalcitrant disease and promote its identification among the ophthalmic community as a real entity that should be addressed or at least considered in the formation of a treatment strategy for all dry eye and meibomian gland disease.

Our study sought to find association with bacterial burden of the eyelid and meibomian glands with signs and symptoms of OSD/meibomian gland dysfunction in a clinic setting. Although our data provide a cross-sectional picture of different demographics, varying presence of bacteria, and a purported link to signs and symptoms of OSD, there are several study limitations that require discussion. First, our small sample size limits the power of our results, and the control group was younger (although this was not by design). When stratifying patients based on OSDI score and meibomian gland dysfunction, it may be expected that this population would be younger. This could have been potentially avoided with a larger sample size or different stratification techniques. However, it is important to note that meibomian gland dysfunction is a progressive, chronic condition, so it is not unexpected that the asymptomatic subjects would be younger. This is a well-known and documented finding in ophthalmic literature, albeit a significant issue for age-match controlling. We feel that the variables undergoing study are not themselves age dependent, but disease dependent. As we found culture positivity to be variable across different degrees of MGD/OSD, we must highlight that these results may be influenced by the significant difference in age between Group A and the other groups, considering MGD tends to increase with age. This could be reevaluated in a larger, perhaps alternatively stratified study. In addition, we evaluated each eye independently to assess laterality as an independent variable. Given that each subject had two eyes, there was no confounding data or inherent bias. Further analysis may involve the averaging of the two eyes; however, we did not find additional utility or novel/unexpected results when doing so. Finally, use of an immunosuppressant (whether topical or other) was not part of our exclusion criteria; therefore, this may have influenced our results as far as bacterial colonization.

\section{Conclusions}

Patients and ophthalmologists alike should understand that meibomian gland dysfunction and related dry eye and OSD are chronic conditions that require regular, if not daily regimens to maintain healthy eyelids and ocular surface. ${ }^{1,4,5,8,11,15}$ Based on our study results and others, we can continue to use our armamentarium of diagnostic tests to guide us in individualizing treatments - but now with the enlightenment that addressing bacterial presence and/or biofilm aspect of these diseases may be of equal (and in some, perhaps, more) importance in providing efficacious treatment.

\section{Acknowledgments}

This research did not receive any specific grant from funding agencies in the public, commercial, or not-forprofit sectors. None of the authors have any financial interests to disclose in relation to this study.

\section{Disclosure}

Dr Alanna Nattis reports grants from Alcon and Glaukos Corp, outside the submitted work. Dr Eric Donnenfeld reports personal fees from Johnson \& Johnson, Blephex, and Sight Sciences, during the conduct of the study and personal fees from Allergan, Alcon, Bausch \& Lomband Novartis, outside the submitted work. The authors report no other conflicts of interest in this work.

\section{References}

1. Pitts J, Lievens C. Put the squeeze on meibomian gland disease. Review of Optometry. 2009. Available from: www.reviewofoptome try.com/article/put-the-squeeze-on-meibomian-gland-disease. Accessed July 5, 2019

2. Albietz J, Lenton LM. Effect of antibacterial honey on the ocular flora in tear deficiency and meibomian gland disease. Cornea. 2006;25 (9):1012-1019. doi:10.1097/01.ico.0000225716.85382.7b

3. Ianaro A, Ialenti A, Maffia P, et al. Anti-inflammatory activity of macrolide antibiotics. J Pharmacol ExpTher. 2000;291(1):156-163. 
4. Yoo S, Lee D, Chang M. The effect of low-dose doxycycline therapy in chronic meibomian gland dysfunction. Korean J Ophthalmol. 2005;19(4):258-263. doi:10.3341/kjo.2005.19.4.258

5. Geerling G, Tauber J, Baudouin C, et al. The international workshop on meibomian gland dysfunction: report of the subcommitte on management and treatment of meibomian gland dysfunction. IOVS. 2011;52(4):2050-2064.

6. Bethke W Putting Dry Eye to the Test. Review of ophthalmology 2014 November. Avaialble from: https://www.reviewofophthalmol ogy.com/article/putting-dry-eye-to-the-test. Accessed June 6, 2019.

7. Arciniega JC, Wojtowicz JC, Mohamed EM, McCulley JP. Changes in the evaporation rate of tear film after digital expression of meibomian glands in patients with and without dry eye. Cornea. 2011;30 (8):843-847. doi:10.1097/ICO.0b013e31820cd291

8. Dougherty J, McCulley J. Comparative bacteriology of chronic blepharitis. Br J Ophthalmol. 1984;68:524-528. doi:10.1136/bjo.68.8.524

9. Dougherty J, McCulley J. Bacterial lipases and chronic blepharitis. Invest Ophthalmol Vis Sci. 1986;27:486-491.

10. Dougherty J, McCulley J, Silvany R, Meyer D. The role of tetracycline in chronic blepharitis: inhibition of lipase production in staphylococci. Invest Ophthalmol Vis Sci. 1991;32(11):2970-2975.

11. Foulks G, Borchman D. Meibomian gland dysfunction: the past, present and future. Eye \& Contact Lens. 2010;36(5):249-253. doi:10.1097/ICL.0b013e3181ef0d37

12. Pult H, Reide-Pult B. Non-contact meibomography in diagnosis and treatment of non-obvious meibomian gland dysfunction. J Optom. 2012;5(1):2-5. doi:10.1016/j.optom.2012.02.003

13. Alsuhaibani A. Carter K Abramoff M, Nerad J. Utility of meibography in the evaluation of meibomian glands morphology in normal and diseased eyelids. Saudi J Ophthalmol. 2011;25:61-66.

14. Olennikov L, Cunningham D, Whitley W Review of Optometry. 2016 May. Available from: https:/www.reviewofoptometry.com/arti cle/improve-your-understanding-of-meibomian-gland-function-anddysfunction. Accessed June 6, 2019.

15. Rynerson J, Perry HD. DEBS -a unification theory for dry eye and blepharitis. Clin Ophthalmol. 2016;10:2455-2467. doi:10.2147/ OPTH.S114674

16. Argudin MA, Vanderhaegen W, Vandendriessche S, et al. Biofilm formation of ica operon-positive Staphylococcus epidermidis from different sources. APMIS. 2015;123(12):1081-1089. doi:10.1111/ apm. 12472

17. Messmer EM. The Pathophysiology, diagnosis and treatment of dry eye disease. Dtsh Arztebl Int. 2015;112(5):71-81.

18. Yeotikar NS, Zhu H, Markoulli M, Nichols KK, Naduvilath T, Papas EB. Functional and morphologic changes of meibomian glands in asymptomatic adult population. Invest Ophthalmol Vis Sci. 2016;57 (10):3996-4007. doi:10.1167/iovs.15-18467

19. Pult H Meibography in Clinical Practice. Ophthalmology Times. 2012. Available from: http://ophthalmologytimes.modernmedicine. com/news/meibography-clinical-practice?page=full. Accessed June 6, 2019. doi:10.1094/PDIS-11-11-0999-PDN

20. Nichols KK, Nichols JJ, Lynn Mitchell G. The relation between tear film tests in patients with dry eye disease. Ophthalmic Physiol Opt. 2003;23(6):553-560.
21. OSDI Questionnaire, Allergan. Available fom: http://www.dryeye zone.com/documents/osdi.pdf. Accessed June 6, 2019.

22. Trofa D, Gacser A, Nosanchuk J. Candida parapsilosis, an Emerging Fungal Pathogen. Clin Microbiol Rev. 2008;21(4):606-625. doi:10.1128/CMR.00013-08

23. Bochler S, Tonning B, Skov R, Prag J. Staphylococcus lugdunensis, a common cuse of skin and soft tissue infections in the community. $J$ Clin Microbiol. 2009;47(4):946-950. doi:10.1128/JCM.01024-08

24. Inada N, Nakashima M, Shoji J. Severe Staphylococcus lugdunesis keratitis. Infection. 2015;43:99-101. doi:10.1007/s15010-014-0669-2

25. Gopal L, Ramaswamy A, Madhavan H, et al. Endophthalmitis caused by acinetobacter calcoaceticus: a profile. Indian J Ophthalmol. 2003;51(4):335-340.

26. Glew RH, Moellering RC, Kunz LJ. Infections with Acinetobacter calcoaceticus (Herellea vaginicola): clinical and laboratory studies. Medicine. 1977;56(2):79-97. doi:10.1097/00005792-197703000-00001

27. Brooke J. Stenotrophomonas maltophilia: an emerging global opportunistic pathogen. Clin Microbiol Rev. 2012;25(1):2-41. doi:10.1128/ CMR.00019-11

28. Mauger T, Kuennen R, Smith R, Sawyer W. Acanthamoeba and Stenotrophomonas maltophilia keratitis with fungal keratitis in the contralateral eye. Clin Ophthalmol. 2010;4:1207-1209. doi:10.2147/ OPTH.S14507

29. Mauger TF, Craig E. Combined acanthamoeba and Stenotrophomonas maltophilia keratitis treated with a conjunctival flap followed by penetrating keratoplasty. Cornea. 2006;25(5):631633. doi:10.1097/01.ico.0000214214.45070.b6

30. Dutkiewicz J, Mackiewicz B, Lemieszek K, Golec M, Milanowski J. Pantoea agglomerans: a mysterious bacterium of evil and good. Part III. Deleterious effects: infections of humans, animals and plants. Ann Agric Environ Med. 2016;23(2):197-205. doi:10.5604/12321966.1203878

31. Lee N, Chung Y, Park J. A Case of Pantoea Endophthalmitis. Korean J Ophthalmol. 2010;24(5):318-321. doi:10.3341/kjo.2010.24.5.318

32. Cheng S, Lin Y, Kuo C, Lai L. Cladosporium keratitis - A case report and literature review. BMC Ophthalmol. 2015;15:106. doi:10.1186/ s12886-015-0092-1

33. Kivanc SA, Kivanc M, Bayramlar H. Microbiology of corneal wounds after cataract surgery: biofilm formation and antibiotic resistance patterns. J Wound Care. 2016;25(1):12,14-19. doi:10.12968/ jowc.2016.25.1.12

34. Hastings JW, Greenberg EP. Quorum sensing: the explanation of a curious phenomenon reveals a common characteristic of bacteria. $J$ Bacteriol. 1999;181(9):2667-2668.

35. Mand P, Eliason J. Blepharitis: overview and classification. In: Mannis M, Holland E, editors. Cornea Fundamentals, Diagnosis and Management. New York(NY): Elsevier; 2017:353-356.

36. Knect LD, O'Connor G, Mittal R, et al. Serotonin activates bacterial quorum sensing and enhances the virulence of Pseudomonas aeruginosa in the host. EBioMedicine. 2016;9:161-169. doi:10.1016/j. ebiom.2016.05.037

37. Uchiyama E, Aronowicz JD, Butovich IA, McCulley JP. Pattern of vital staining and its correlation with aqueous tear deficiency and meibomian gland dropout. Eye Contact Lens. 2007;33(4):177-179. doi:10.1097/01.icl.0000253054.10349.2f
Clinical Ophthalmology

\section{Publish your work in this journal}

Clinical Ophthalmology is an international, peer-reviewed journal covering all subspecialties within ophthalmology. Key topics include: Optometry; Visual science; Pharmacology and drug therapy in eye diseases; Basic Sciences; Primary and Secondary eye care; Patient Safety and Quality of Care Improvements. This journal is indexed on PubMed
Central and CAS, and is the official journal of The Society of Clinical Ophthalmology (SCO). The manuscript management system is completely online and includes a very quick and fair peer-review system, which is all easy to use. Visit http://www.dovepress.com testimonials.php to read real quotes from published authors. 